\title{
Observations sur le comportement migratoire des Aloses (Alosa alosa L.) dans le canal artificiel de l'usine de Golfech
}

\author{
A. Belaud 1 \\ R. Dautrey' \\ R. Labat 1 \\ J.P. Lartigue 1 \\ P. $\operatorname{Lim} 1$
}

Mots-clés : Poissons migrateurs, Alose, Alosa alosa L., Fleuve Garonne.

Pendant quatre saisons de migration successives, il a été procédé à des captures d'aloses (Alosa alosa L) bloquées devant l'usine hydroélectrique de Golfech. Cet article rapporte les observations sur le comportement migra toire de ces poissons dans le parcours artificiel constitué par le canal de restitution.

La migration débute dès lors que la température de l'eau atteint $16^{\circ} \mathrm{C}$ environ et, au-dessus de ce seuil, l'abondance des aloses est maximale en période de réchauffement de l'eau, tandis que la migration s'interrompt en phases de refroidissement.

La relation température de l'eau - abondance des prises est significative en début de migration et disparait des que la migration a atteint son développernent maximal.

Parallèlement au facteur thermique, les facteurs hydrauliques (débit turbiné, còte aval, répartition de l'eau sur les turbines, dégrillages...) déterminent la fréquentation du canal par les aloses.

Les expériences d'évaluation du stock par des techniques de marquage échouent en raison du taux infime de recaptures (imputable au stress).

L'analyse des cahiers de pêche démontre que les aloses ne s'accumulent pas d'un jour à l'autre devant l'usine.

Les diverses pêches (différant par les engins et les lieux) indiquent que les aloses explorent le canal de restitution par des mouvements de va-et-vient, transitant par le fond du canal et se repliant, la nuit ou le jour quand les conditions sont défavorables, dans les eaux profondes de la Garonne et de la partie aval du canal.

Par rapport aux observations rapportées antérieurement et concernant des cours d'eau naturels, les présents résultats font apparaître un comportement typique d'un segment artificiel.

Observations on the migration behaviour of Alewive (Alosa alos $a$.) in the artificial canal of Golfech hydroelect ric plant Keywords : Fish migration, Alewive, Alosa alosa L., Garonne river.

For 4 consecutive years, daily fishing over the period of upstream migration of alewives (Alosa alosa $\mathrm{L}$.) were performed on the site of Golfech : an hydroelectric plant on the Garonne river. This paper presents observations on the migratory behaviour of this fish in the artificial canal located between the plant and the confluence.

The migration develops in spring when the water temperature reaches approximately $16^{\circ} \mathrm{C}$ and, above this level, the maximal affluence of alewives corresponds with increasing temperature phases.

The relationship between water temperature and capture abundance is significant if one consider the beginning of the migration, then becomes inconsistent when the migration reaches the maximum.

Similar to the thermal factor, there are hydraulic characteristics which influence the alewive migration (water flow through the different turbines, downstream level...).

The evaluation of the fish stock, using marking techni is not satisfactory because of the small number of fish recaptured. Analysis of daily fishing data demonstrates that there is no accumulation of alewives day after day, in front of the factory.

1. Laboratoire d'Ichtyologie Appliquée, E.N.S.A.T., 145 Avęrue de Muret, 31076 Toulouse Cédex, France. 
The various fishing operations indicate that alewives explore diurnally upward and downward the artificial canal by passing on the botton. Every night or when the conditions are unfavourable they stay in deep water downstream in the river.

Compared with previous observations the actual results indicate a typical migratory behaviour in an artificial canal.

\section{Introduction}

Depuis 1980, le Laboratoire d'Ichtyologie Appliquée de l'E.N.S.A.T procède, en collaboration avec le Conseil Supérieur de la Péche (C.S.P. Délégation Midi-Pyrénées) et les Fédérations Départementales de Pêche riveraines, pour le compte d'Electricité de France (Région Equipement Marseille) à la capture d'aloses (Alosa alosa L.) au pied de l'usine de Golfech dans le canal artificiel de restitution.

Le but principal de ces opérations est de transporter des géniteurs en amont pour tenter de recoloni. ser la Garonne entre Golfech et Toulouse et le Tarn jusqu'au prochain barrage de Sainte-Livrade : zones autrefois riches en frayères à aloses. De 248 en 1980 . les captures sont passées à 2503 en 1981, à 6363 en 1982 et à 7227 en 1983. A la suite de ces opérations de transfert, des preuves de fraie en amont de Golfech ont été obtenues et la capture, chaque année aux alentours de septembre, d'alosons en cours de dévalaison, témoigne de l'efficacité des opérations.

Ces travaux de protection d'une espèce en voie de régression en Garonne donnaient l'occasion d'étu. dier certains aspects du comportement migratoire. Le présent article expose ces résultats.

L'étude du comportement migratoire des aloses revêtait un intérêt fondamental car les données bibliographiques les plus completes concernent principalement l'alose américaine Alosa sapidissima (Leggett \& Whitney 1972, Talbot 1953), études effectuées sur 32 et 13 années, respectivement).

Il existe aussi des renseignements plus ponctuels mais qui se rapportent soit encore à l'alose américaine (Walburg \& Nichols 1967, Carscadden \& Leggett 1975, Legget 1976, Chittenden 1976) soit, en France, à l'alose du Rhône (Alosa fallax) aujourd'hui presque disparue (Quignard \& Kartas 1977; Rameyre \& al. 1976). Pour la grande alose Alosa alosa L., les travaux de Raveret-Wattel (1894), C.T.G.R.E.F. (1979), Cassou-Leins \& Cassou-Leins (1981), Douchement (1981) et Maudet (1983) fournissent des indications précieuses sur les périodes de migration, l'écobiologie, la biométrie et l'halieutique. Dans ces travaux antérieurs, faute de moyens d'investigation appropriés, les divers aspects du comportement migratoire de la grande alose ne sont qu'effleurés.

Les observations sur le comportement de la grande alose à Golfech revêtent un intérêt appliqué puisque se pose le problème de la construction d'un dispositif de franchissement automatique de la dénivellation de l'usine. De ce point de vue, les travaux antérieurs ne concernent que des cours naturels de rivières, exceptés les articles de Talbot (1953) et de Saila \& al. (1972) qui rapportent des observations sur le comportement des aloses américaines au voisinage d'une " passe à poissons". Les observations faites à Golfech à l'occasion des opérations de capture et de transfert des aloses bloquées seront précieuses pour les aménagistes.

\section{1. - Le site de Golfech}

Un barrage sur la Garonne, situé à Malause, dérive de l'eau dans un canal artificiel de $10,6 \mathrm{~km}$ jusqu'à la chute de l'usine hydroélectrique de Golfech, mise en service en 1973. L'eau turbinée sort dans un canal de restitution qui rejoint le cours naturel de la Garonne à Lamagistère, 1850 mètres en aval de l'usine (fig. 1).

Le canal de restitution a une section trapézoïdale : sa largeur est de 40 mètres au fond et de 60 mètres en surface, sa profondeur moyenne est de 5 mètres, excepté au pied de l'usine où la profondeur est de 10 mètres.

L'écoulement est très tourmenté à la sortie des trois groupes « Kaplan * de l'usine, puis plus régulier à partir des 100 premiers mètres.

La figure 2 est un plan de la zone proche de l'usine. La sortie des trois turbines est recouverte d'une dalle de beton formant une plateforme praticable pour la pêche lorsque la côte aval est inférieure à 48,60 mètres. La figure 2 montre aussi comment lé canal s'élargit, en constituant de chaque côté une zone d'eaux plus calmes et où on peut observer généralement un courant de retour. 


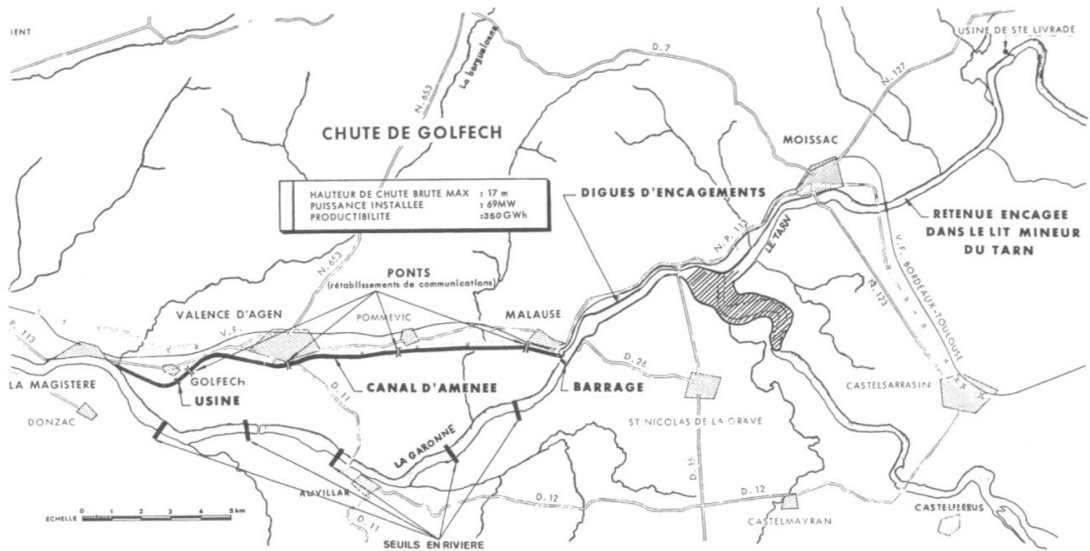

Fig. 1. Situation générale et disposition des aménagements.

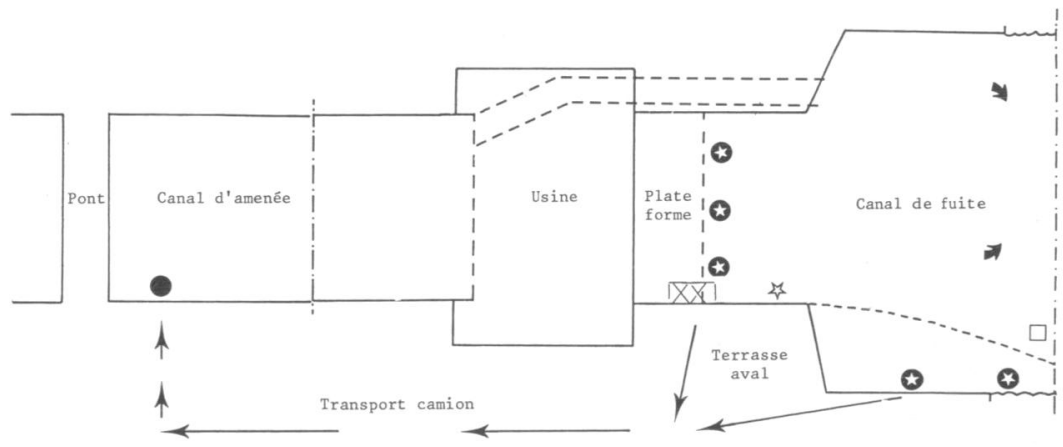

4 Lieu de capture au "coule"

Point de déversement amont

7 Lieu de pêche à la nasse

[ Lieu de pêche au barro

1 Lieu de pêche au filet flottant dérivant

Fig. 2. Croquis de la zone proche de l'usine avec indication des points d'activité de pêche et de déversement. 


\section{2. - I.e fonctionnement de l'usine}

L'usine peut recevoir $3 \times 180 \mathrm{~m}^{3}$ par seconde. Les débits moyens de la Garonne à Lamagistère, calculés entre 1941 et 1981 sont indiqués dans le graphe de la figure 3.

Trois situations peuvent être distinguées :

\section{- Situation I}

En période estivale et en automne, toute l'eau de Garonne passe par l'usine exceptés $7 \mathrm{~m}^{3} / \mathrm{s}$ de débit réservé à Malause.

\section{- Situation 2}

En périodes de forts débits, l'usine n'absorbe qu'une partie du débit de Garonne qui se répartit entre le canal et le "bras mort " de la Garonne.

\section{- Situation 3}

En période de crue, l'usine cesse de fonctionner et le barrage de Malause est abaissé. Toute l'eau passe par le "bras mort".
Les recherches exposées dans cet article ont été faites en majorité en situation 1, rarement en situation 2.

\section{3. - Techniques}

- Captures au " coule "

La première année a été consacrée à la mise au point d'une méthode de pêche efficace et représentative de l'abondance des poissons. Après des essais de carrelet, de senne, de verveux, la technique retenue par la suite fut la pêche " au coule".

Le " coule" se présente comme une grande épuisette munie d'un filet de 1,30 mètre de diamètre et de 80 centimètres de profondeur, en maille de 30 millimètres. Il est manœuvré par un long manche. La pèche a été réalisée par la mème personne depuis 1980 et les mêmes points de pêche ont été exploités (fig. 2).

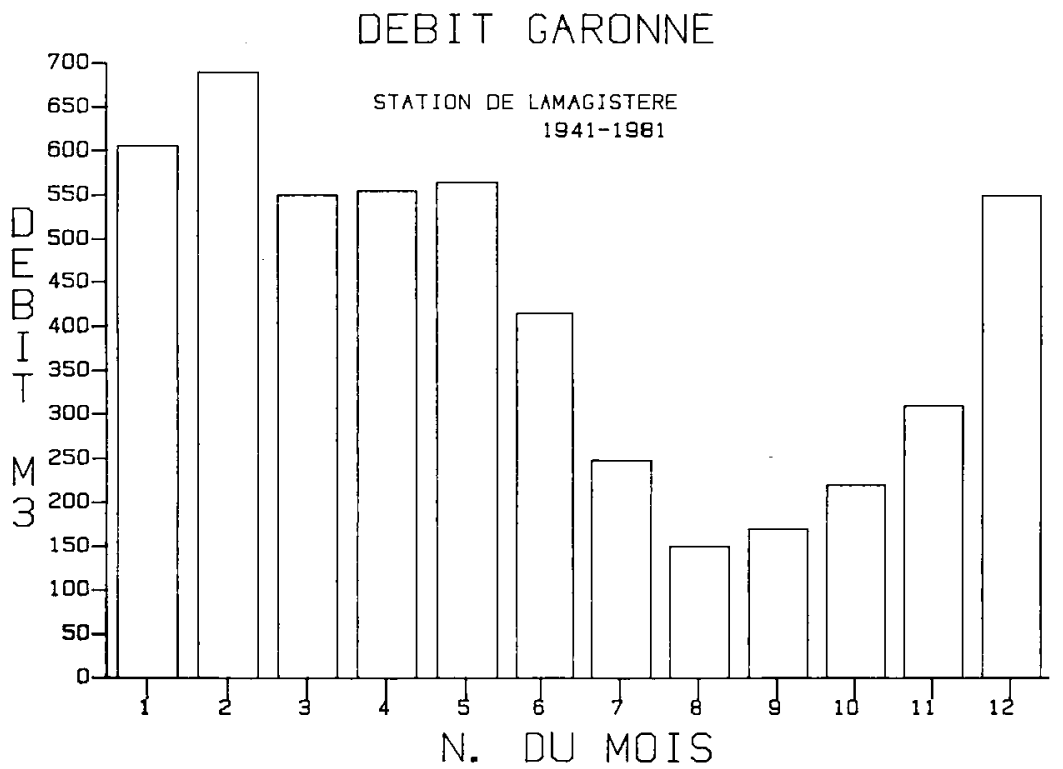

Fig. 3. Représentation graphique des débits moyens mensuels de la Garonne, étudiés à Lamagistère entre 1941 et 1981. 
En période de fortes eaux (cote aval supérieure à 48,50 mètres), le pêcheur procède depuis un ponton installé en rive droite au-dessus de la surface de l'eau. Il procède soit par sondage de l'eau, à 1,50 à 2 mètres au-dessous de la surface entre la sortie de la première turbine et le mur rive droite (pêche de type " $S$ ") soit à " coule "fixe sur la plateforme de béton qui recouvre la sortie des turbines (pêche de type « F n) (fig. 2).

Dès que la cote aval le permet, le pècheur se déplace sur la plateforme pour aller sonder succes. sivement les zones d'eau calme situées au voisinage de la sortie des trois turbines. (La violence du courant ne permet pas de mancuvrer le a coule "face à la sortie des turbines mais la pêche est possible dans les eaux plus calmes situées entre deux turbines ou entre une turbine et un mur de rive).

Occasionnellement, la pêche au "coule" a èté effectuée plus en aval de l'usine au niveau des plans inclinés (rive droite et rive gauche) de la partie élargie du canal (fig. 2).

L'unité d'effort de pêche au "coule " a été définie arbitrairement comme une heure de manipulation de l'appareil (type " $S$ n et type " $F$ " confondus).

- Autres moyens de pèche (fig. 2)

Pour compléter les résultats, il a été procédé, occa. sionnellement, à des pèches à l'aide d'engins différents du " coule ".

En 1982, il a été pêché près du mur rive droite a l'aide de deux nasses: la première de $2,3 \times 1,5 \times$ 1,3 mètres et ensuite une grande nasse de $6 \times 3 \times$ 3 mètres. L'utilisation, en 1982, d'un " barro " (filet tournant mu par le courant) a démontré l'inadéquation de ce moyen de peche traditionnel dans le canal artificiel de Golfech.

En 1983, deux journées ont été consacrées à la péche au filet flottant dérivant, tendu sur toute la longueur du canal. La profondeur du filet était de 2 mètres.

\section{- Contrôle des caractéristiques de l'eau}

En 1980, 1981 et 1982, seules la température de l'eau et la cote aval de l'usine furent relevées. En 1983, les valeurs de temperature, $\mathrm{pH}$, conductivité, oxygène et turbidité ont été contrôlées deux fois par jour à l'aide d'un appareil de terrain multifonctions HORIBA U.7. Malheureusement, une panne de la voie " turbidité " nous a privés de cette précieuse indication pendant une grande partie de la campagne.
En plus des lectures d'échelle limnigraphique au pied de l'usine, les responsables de la production E.D.F. nous ont permis, en 1983, de consulter les enregistrements, effectués en continu, des valeurs de débit turbiné.

\section{- Expériences de marquage}

Les premières années, les expériences de marquage furent réalisées à l'aide d'un pistolet marqueur de vêtements délivrant des bagues en plastique avec un arrêt en " $T$ ". Ces marques étaient introduites dans les muscles dorsaux au niveau de la nageoire dorsale. Aucune marque ne fut retrouvée en 1980 et 1981 .

En 1983, il a été utilisé des bagues métalliques numérotées et serties soit sur la nageoire caudale, soit sur la nageoire dorsale à l'aide d'une pince spéciale. 594 aloses ont ainsi été marquées en 1983.

\section{4. - Résultats}

Les pêches les plus abondantes, permettant le transfert massif des géniteurs en amont de l'usine, ont été réalisées au "coule "et en rive droite (ponton et plate-forme) (fig. 2).

\section{1. - Résultats des pêches au " coule"}

L'anné 1980 a donné un nombre d'aloses insuffisant pour réaliser une étude quantitative de la migration.

Pour les années 1981, 1982 et 1983, les résultats sont reportés dans les tableaux I, II et III : température de l'eau, débit de la Garonne, nombre d'aloses capturées, temps de pêche et densité des prises (nombre d'aloses prises rapporté au temps de pêche exprimé en heure).

L'année 1983 a donné lieu à des pointages plus précis portant sur le temps, l'oxygène, la turbidité, le $\mathrm{pH}$ de l'eau, la cote en aval de l'usine, le type de pêche ( $S$ ou $F$ ) ainsi que le détail de la répartition des captures dans la journée. Le tableau III permet une analyse complète des résultats :

- Mois de mai 1983

Le temps froid est responsable de la faible montée des aloses. De plus, la pêche n'a pu être réalisée que depuis le ponton rive droite.

Les meilleures journées sont le samedi 21 et le mardi 31 où les prises ont dépassé 12 aloses à 
Tableau I. Résultats des péches aux alosés pendan! la saison 1981. Puur chaque journée de pêche, i] a été com posé une ligne indiquant la date, la temperature de l'eau, le débit du fleuve, puis les captures (capture totale " Capt ; la durée des activités de péche pour la journée et la densité des prises, c'est-a-dire le nombre moyen d'aloses pris par heure de peche)

\begin{tabular}{|c|c|c|c|c|c|}
\hline $\begin{array}{l}14-5 \\
15-5 \\
16-3 \\
17-5 \\
19-5 \\
19-5 \\
20-5 \\
21-5 \\
22-5 \\
23-5 \\
24-5 \\
25-5 \\
26-5 \\
27-3\end{array}$ & 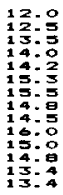 & $\begin{array}{l}950 \\
815 \\
710 \\
635 \\
570 \\
355 \\
545 \\
555 \\
625 \\
625 \\
540 \\
540 \\
730 \\
730\end{array}$ & $\begin{array}{r}0 \\
1 \\
5 \\
2 \\
8 \\
10 \\
25 \\
20 \\
26 \\
189 \\
197 \\
27 \\
1 \\
1\end{array}$ & $\begin{array}{l}4.00 \\
4.30 \\
7.00 \\
5.90 \\
5.00 \\
6.00 \\
6.45 \\
8.00 \\
6.30 \\
7.15 \\
6.90 \\
3.00 \\
3.00\end{array}$ & 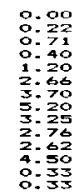 \\
\hline $\begin{array}{r}1-6 \\
2=6 \\
3=6 \\
3-6 \\
6-6 \\
7=6 \\
9-6 \\
9=6 \\
10-6 \\
11=6 \\
12-6 \\
13=6 \\
13-6 \\
10-6 \\
17-6 \\
19-6 \\
19=6 \\
20-6 \\
22-6 \\
23-6 \\
24-6 \\
25-6 \\
26-6 \\
27-6 \\
29-6 \\
30-6\end{array}$ & 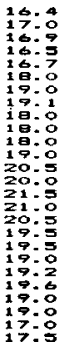 & $\begin{array}{l}520 \\
505 \\
560 \\
625 \\
300 \\
462 \\
453 \\
489 \\
509 \\
491 \\
436 \\
431 \\
392 \\
369 \\
369 \\
352 \\
319 \\
296 \\
237 \\
237 \\
221 \\
214 \\
239 \\
239 \\
595 \\
370\end{array}$ & 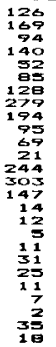 & $\begin{array}{l}9.15 \\
7.30 \\
3.30 \\
7.45 \\
6.00 \\
6.30 \\
6.00 \\
7.30 \\
6.30 \\
7.00 \\
7.00 \\
7.00 \\
6.00 \\
6.13 \\
4.00 \\
3.30 \\
3.00 \\
6.45 \\
6.00 \\
6.00 \\
6.30 \\
6.00 \\
4.00 \\
6.45 \\
6.30\end{array}$ & 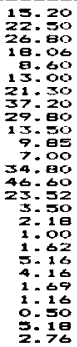 \\
\hline $\begin{array}{r}2=7 \\
2-7 \\
3-7 \\
4=7 \\
6-7 \\
9-7 \\
9-7 \\
10-7 \\
12-7 \\
13-7\end{array}$ & 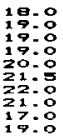 & $\begin{array}{l}403 \\
333 \\
313 \\
339 \\
270 \\
291 \\
256 \\
469 \\
520 \\
364\end{array}$ & $\begin{array}{l}10 \\
58 \\
37 \\
25 \\
30 \\
191 \\
48 \\
16 \\
11\end{array}$ & $\begin{array}{r}4.00 \\
4.00 \\
4.30 \\
4.00 \\
4.00 \\
6.00 \\
7.00 \\
6.30 \\
6.00\end{array}$ & $\begin{array}{l}2.50 \\
1.50 \\
3.70 \\
1.50 \\
6.75 \\
2.00 \\
2.20 \\
0.40 \\
1.92\end{array}$ \\
\hline \multicolumn{6}{|c|}{ $\pm * *$} \\
\hline
\end{tabular}

I'heure. Le facteur température ne semble pas mis en jeu pour expliquer cela. Par contre, les enregis. trements de débil de la centrale indiquent que, ces jours-là, le niveau d'eau à l'aval de l'usine a progressivement augmenté. L'accroissement du niveau aval apparait donc comme un facteur favorable.

La journée du 24 mai, marquée par un niveau constant, a aussi donné lieu à des prises substantielles.

Pour la journée du 20 mai, aucune relation n'a pu être établie entre le nombre élevé de prises et les facteurs hydrauliques relevés à Golfech (sans doute la température de $15,4^{\circ} \mathrm{C}$ a-t-elle déclenché le mouvement des aloses?).
Tableau 11. Résultats des pèches aux aloses pendant la sai. son 1982 (méme présentation que pour le Tableau I).

\begin{tabular}{|c|c|c|c|c|c|}
\hline \multicolumn{2}{|c|}{ PECHES AU } & \multicolumn{3}{|c|}{ "COLEE" 1982} & \multirow[b]{2}{*}{$\begin{array}{r}22.90 \\
26.60 \\
13.60 \\
23.10 \\
29.70 \\
17.90 \\
7.80 \\
9.40 \\
16.90 \\
31.90 \\
13.60\end{array}$} \\
\hline $\begin{array}{l}27-5 \\
18-5 \\
19-5 \\
21-5 \\
22-5 \\
23-5 \\
24-3 \\
25-5 \\
26-5 \\
27-5 \\
29-5\end{array}$ & $\begin{array}{l}18-5 \\
17: 3 \\
16: 3 \\
16=4 \\
16: 5 \\
16: 3 \\
16: 3 \\
16: 5 \\
17=0 \\
17=0 \\
17=5\end{array}$ & $\begin{array}{l}332 \\
346 \\
349 \\
335 \\
323 \\
323 \\
317 \\
323 \\
302 \\
311 \\
323\end{array}$ & $\begin{array}{r}132 \\
193 \\
90 \\
164 \\
139 \\
112 \\
64 \\
61 \\
127 \\
213 \\
69\end{array}$ & $\begin{array}{l}5.45 \\
7.15 \\
5: 55 \\
7.05 \\
5.30 \\
6.150 \\
6.30 \\
5: 30 \\
7.25 \\
6.00\end{array}$ & \\
\hline $\begin{array}{l}1-6 \\
2-6 \\
3-6 \\
4-6 \\
5-6 \\
6-6 \\
7=6 \\
8-6 \\
9-6 \\
10-6 \\
11-6 \\
13-6 \\
13-6 \\
13-6 \\
13-6 \\
16-6 \\
17-6 \\
19-6 \\
19-6 \\
20-6 \\
21-6 \\
22-6 \\
23-6 \\
24-6 \\
25-6 \\
26-6 \\
29-8 \\
29-6 \\
30-6\end{array}$ & 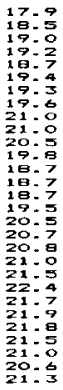 & $\begin{array}{l}295 \\
274 \\
241 \\
259 \\
311 \\
246 \\
254 \\
264 \\
237 \\
244 \\
254 \\
297 \\
320 \\
292 \\
299 \\
274 \\
269 \\
249 \\
249 \\
226 \\
202 \\
200 \\
147 \\
195 \\
179 \\
162 \\
162 \\
137 \\
137\end{array}$ & 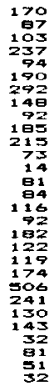 & 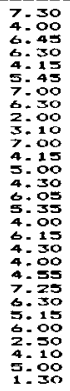 & 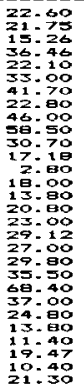 \\
\hline $\begin{array}{l}1=7 \\
2-7 \\
3=7 \\
4=7 \\
6-7 \\
9=7 \\
9=7 \\
10=7 \\
112=7 \\
12-7\end{array}$ & 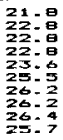 & $\begin{array}{l}144 \\
131 \\
131 \\
135 \\
135 \\
121 \\
110 \\
90 \\
107 \\
95\end{array}$ & $\begin{array}{r}69 \\
60 \\
174 \\
95 \\
117 \\
107 \\
107 \\
50 \\
65 \\
15\end{array}$ & $\begin{array}{l}3.15 \\
2.150 \\
2.50 \\
3.00 \\
4.10 \\
3.00 \\
5.00 \\
2.15 \\
2.15 \\
3.00\end{array}$ & $\begin{array}{r}20.90 \\
20.70 \\
69.50 \\
28.30 \\
29.25 \\
5.60 \\
21.40 \\
20.80 \\
20.90 \\
1.00\end{array}$ \\
\hline & & & & & \\
\hline
\end{tabular}

Les autres jours du mois de mai n'ont permis que des prises éparses.

En période d'initiation de migration et par temps frais, les aloses viennent certains jours ou le niveau du canal ne baisse pas, faire des incursions au pied de l'usine. L'irrégularité des prises traduit l'intermittence de l'activité migratoire dans le canal. Il n'y a pas accumulation massive des aloses au pied de I'usine méme au lendemain des jours d'interruption de pêche.

\section{- Mois de juin 1983}

Les températures dépassent $16^{\circ} \mathrm{C}$ tout le mois, alors que cette valeur $n$ 'avait pas été at teinte en mai. La fréquence des captures est élevée (jusqu'à 72 alo. ses à l'heure). 
Tableau III. Résultats des péches aux aloses pendant la sajson 1983 (même présentation que dans le Tableau I).

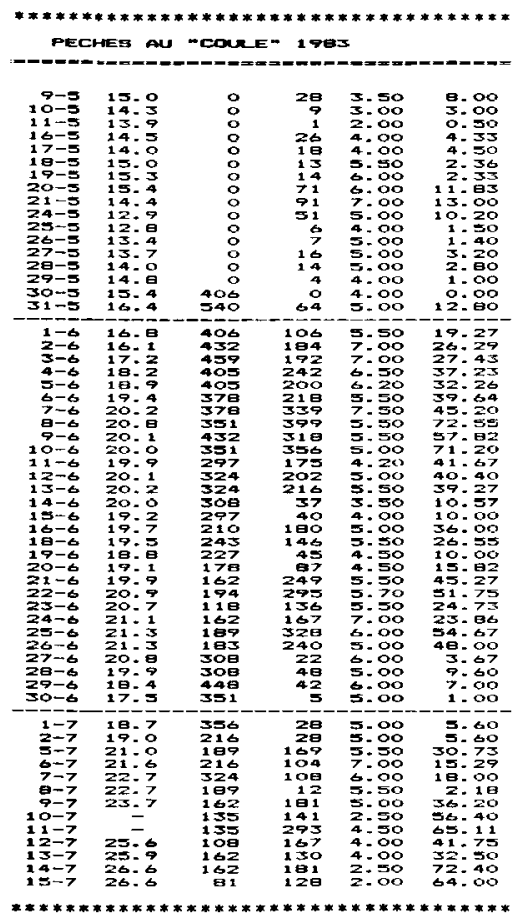

Les phases de réchauffement des eaux (du $1^{\text {er au }}$ 13 juin, mais aussi du 20 au 22 juin) correspondent à de fortes captures; les phases de refroidissement (du 14 au 20 juin et surtout à partir du 26 juin) pro. voquent une raréfaction des aloses. Bien que les captures soient en rapport avec la température et l'évolution thermique, d'autres facteurs peuvent interférer au jour le jour :

le 18 juin donne d'assez bons résultats malgré le refroidissement, vraisemblablement en rapport avec le type de fonctionnement régulier de l'usine ce jour-là ; les conditions hydrauliques à la sortie des turbines peuvent contrarier la péche et entrainer des variations dans les captures d'une heure à l'autre. Ceci est difficile à étudier en ce mois de juin où :

1. les conditions de turbinage ont varié à l'infini en phase de baisse du débit du fleuve,

2. le pecheur a adapté sa technique au fil des heures pour s'affranchir de ces variations d'écoulement et,

3. le facteur thermique et le facteur date favoriseraient la remontée des aloses.

Il faut retenir le róle néfaste des fluctuations de niveau rapides et des changements fréquents de turbine ainsi que l'effet contrariant des opérations de dégrillage (évacuation des débris flottants à la grille de l'usine).

- Mois de juillet 1983

Exceptés les 1, 2 et 8 juillet, les prises sont restées nombreuses, atteignant le rythme record de 72,4 prises à l'heure le 14 juillet.

Les faibles prises des 1 et 2 juillet prolongent les observations identiques de la fin juin et s'expliquent par un refroidissement de l'eau à partir du 26 juin. A partir du 5 juillet, la température de l'eau remonte sans interruption jusqu'au 15 juillet et des captures se maintiennent à un niveau élevé jusqu'à cette date. La période de pêche a cessé avant la fin de la migration mais la température de l'eau $\left(26^{\circ} \mathrm{C}\right)$ et la canicule ne permettaient plus de transférer les poissons dans de bonnes conditions.

. Pour expliquer les résultats médiocres du 8 juillet ( 12 prises en 5 h 30 de pêche), la température et le temps ne paraissent pas en jeu (semblables à la veille ou au lendemain) mais les eaux étaient boueu. ses (orages de l'avant-veille) et le fonctionnement de l'usine a nécessité des dégrillages fréquents. En outre, il y a eu ce jour-là de fréquents changements de répartition de l'eau sur les turbines, autant de facteurs qui découragent les aloses pour remonter dans le canal de Golfech.

L'arrêt des 3 et 4 juillet ne donne pas lieu à une accumulation d'aloses car les prises abondantes du 5 sont bien réparties dans la journée.

Én période d'abondance des aloses, la pêche sur le plan incliné rive droite permet de capturer des aloses au " coule " et presque autant que lorsqu'on pêche à la sortie des turbines. 


\section{2. - Résultats complémentaires}

En complément des résultats consignés dans les tableaux, il a été remarqué que le "coule " permet souvent des prises multiples, c'est-à-dire que, après plusieurs sondages infructueux, il est fréquent de ramener plusieurs aloses d'un meme coup (record de 17 aloses à la fois !).

A l'observation directe, $i$ l est très rare d'apercevoir des aloses dans les couches d'eau superficielles : ceci ne fut jamais le cas dans la portion de canal "élargie " (fig. 2). Près de l'usine, des aloses peuvent êtré soulevées par les remous et apparaitre furtive. ment près de la surface.

Les pêches au filet flottant dérivant n'ont permis aucune capture d'alose (sur la couche superficielle de 2 metres). La pêche au filet n'est pas possible dans le fond du canal, encombré de débris divers.

Les jours où la pêche est fructueuse, on peut aussi capturer au "coule " des aloses à 100 mètres en aval de l'usine, mais en plus faible nombre par rapport à la pêche au coule au pied de l'usine. En revanche, les jours où il n'y a pas d'aloses à la sortie des tur. bines, il n'y en a pas, non plus, en aval.

Les nasses fixes ont permis de capturer, en 1982. environ 800 aloses. Malgré les améliorations succes. sives (emplacement, nature du filet de revetement, temps d'immersion, profondeur de pèche...), les nasses fixes sont toujours restées moins efficaces que la pèche en mouvement du " coule".

Les expériences de marquage, en 1983, ont porté sur 594 aloses : 66 capturées marquées et relâchées en aval de l'usine ; 228 relâchées en amont de l'usine et enfin, lé 17 juin, 300 aloses capturées à Agen en aval du barrage de Beauregard el relachées en amont de ce barrage d'Agen, situé à $18 \mathrm{~km}$ en aval de l'usine de Golfech.

Seules $\mathbf{4}$ aloses marquées ont été retroùvées. Une alose marquée le 9 mai et reláchée en aval de l'usine a été reprise le 16 juin à Saint.Sixte, c'est-à-dire $4 \mathrm{~km}$ en aval du point de marquage, dans la Garonne. Une alose femelle marquée et transférée à Golfech le 20 mai fut reprise le 16 juillet: ce poisson avait pondu. Enfin, 2 aloses capturées a Beauregard le 17 juin furent reprises: I'une le 26 juin a Agen, c'està-dire $2 \mathrm{~km}$ en aval et l'autre à Golfech le 23 juin, ce qui permet d'évaluer lo lemps de migration AgenGolfech : $18 \mathrm{~km}$ en 5 jours. Ces faibles reprises ne peuvent s'expliquer que par le stress des aloses pen- dant le marquage, provoquant ensuite un comportement anormal (dévalaison, mortalité).

\section{5. - Discussion}

\section{1. - Abondance des aloses susceptibles de se pré- senter à Golfech}

Aprés leur phase de développement en mer, les aloses en état de maturation sexuelle s'engagent dans les fleuves. L'effectif qui entre ainsi en Garonne est difficile à connaît re car l'insuccès des opérations de marquage ne permet pas une étude quantitative. Seule une vaste enquête, le long du segment encore fréquenté par les aloses, permettrait une évaluation de la population de poissons migrateurs. Actuellement, nous ne disposons que de quelques éléments fragmentaires. Ainsi, à titre indicatif, les pointages de Cassou-Leins et Cassou-Leins (communication personnelle) permettent d'estimer à 30000 aloses la fréquentation de la seule frayère d'Agen (en 1982 comme en 1983). Compte-tenu qu'il existe, en aval de Golfech, 4 frayères recencées (Lamagistère, SaintSixte, Agen et Aiguillon), on peut évaluer à 120000 le nombre de géniteurs. A ces chiffres, il convient d'ajouter les captures: 460 tonnes dans l'estuaire de la Gironde en 1977 (C.T.G.R.E.F. 1979) et 50 tonnes uniquement pour la pêcherie d'Agen. La Garonne reçoit donc plusieurs centaines de milliers d'aloses dont certaines tentent de remonter en amont de Golfech (on trouvait, au siècle dernier, des aloses jusqu'à Toulouse mais aussi dans le Tarn (Cassou-Leins \& Cassou-Leins 1981)).

Le principal obstacle du developpement de la migration jusqu'à Golfech - hormis les activités de pêche - est le barrage d'Agen "Beauregard " ( 2 mètres de chute pour un débit de $200 \mathrm{~m}^{3 /} \mathrm{s}$ ). Ce barrage filtre le passage des aloses de manière plus ou moins sélective selon le débit de la Garonne et selon la température, le sexe, la taille... c'est-à-dire selon les facteurs affectant la performance natatoire des poissons (Maudet 1983).

On peut ainsi observer chaque saison des bancs d'aloses en attente devant l'obstacle. Les aloses qui franchissent ce barrage le font généralement en rive droite, en suivant un passage étroit abrité par les aspérités du mur de rive. Les années où le débit est élevé, les aloses peuvent franchir le barrage sur toute sa largeur. Il est donc difficile d'avancer un 
pourcentage d'aloses qui franchit le barrage. Les faits connus sont que :

1. chaque année, il y a des aloses à Golfech, ce qui montre que le pourcentage n'est pas nul et que.

2. la fréquentation de la frayère de Lamagistère (en amont du barrage) est comparable à celle de la frayère d'Agen (en aval) (Cassou-Leins \& Cassou-Leins, communication personnelle). Il peut être considéré, pour la suite de cette dis. cussion, que le barrage d'Agen ("Beauregard "), bien que faisant une sélection, est franchissable pour des milliers d'aloses 2 .

A la réunion du canal de fuite de Golfech et du cours naturel de la Garonne, les aloses rencontrent, généralement (type de fonctionnement $n^{\circ} 1$ de l'usine), un flux plus attractif dans le canal et s'engagent majoritairement dans le canal, délaissant le "bras mort ". Ceci confirme les observations de Rameyre \& al. (1976) sur l'alose du Rhône qui préfère le canal de restitution de l'usine de Vallabrègues au Rhône court-circuité. Le cas de l'alose n'est donc pas différent de ce qui est classiquement admis pour les salmonidés (Weaver 1963).

Pour les aloses qui auraient choisi le "bras mort ", divers obstacles et enfin le barrage de Malause arrêtent la migration.

\section{2. - Profondeur du trajet migratoire dans le canal de Golfech}

A l'observation, peu d'aloses peuvent être aperçues en surface du canal de Golfech, et ceci même les jours de grande affluence démontrée par la pêche. Lorsqu'elles sont aperçues, elles le sont furtivement, au pied de l'usine, dans la zone où les remous très instables désorganisent la nage des poissons. Ainsi, de temps à autre, des aloses sont " soulevées" vers la surface et parfois sont déportées sur la dalle de béton qui recouvre la sortie des turbines. Des aloses, malmenées par ces écoulements violents, se replient parfois en longeant le mur rive droite pour s'abriter dans les eaux plus calmes de l'élargissement du canal (fig. 2). Les observations d'aloses se repliant en longeant le mur rive

2. Ce barrage d'Agen fait actuellement l'objet d'un projet de construction d'une * passe a poissons ". En attendant, la D.D.E. d'Agen a fait procéder en octobre 1983 à un arrangement de blocs d'empierrement, formant une passe rustique. droite sont nombreuses (il est vrai que, compte-tenu des accès, ce fut là le point d'observation le plus fréquenté). Il est probable que, du fait de la symétrie de l'usine, il en soit de même en rive gauche, du moins en dehors des phases de dégrillage et d'évacuation des débris flottants par le canal de défeuillage.

En dehors de ces conditions de nage désorganisée, les aloses semblent préférer les eaux profondes.

Il n'a jamais été observé d'aloses dans la partie aval du canal et, de plus, les pêches au filet dérivant flottant n'ont jamais permis d'en intercepter sur la bande superficielle de 2 metres. Ceci indique que les aloses remontent le canal en profondeur, où du fait de la turbidité, la visibilité est faible. La preuve directe de ceci n'a cependant pas pu être apportée car, pour pêcher au filet au fond du canal, on se heurte à de nombreux débris.

Le fait que les pêcheurs qui pratiquaient la pêche au " coule " dans le canal (avant 1982 où elle fut interdite) manœuvraient le filet à des profondeurs de 1,50 à 2,50 mètres est un argument indirect en faveur du transit des aloses en eaux profondes.

\section{3. - Fluctuations de l'affluence des aloses au pied de l'usine}

Il pouvait a priori être supposé qu'un obstacle comme celui de l'usine de Golfech pouvait occasionner une accumulation durable d'aloses, comme cela s'observait depuis plusieurs années devant le seuil de "Beauregard " (Cassou-Leins \& Cassou-Leins 1981). Cette hypothèse de départ négligeait les différences entre un parcours naturel de rivière et un segment artificiel. L'expérience montre qu'à Golfech, les aloses sont tantôt abondantes tantôt absen. tes au pied de l'usine. La fréquentation peut varier selon les jours mais aussi selon les heures.

Ce résultat conduit à considérer que les aloses ne s'accumulent pas de manière durable mais procèdent par " raids de reconnaissance "suivis de replis plus en aval. A l'appui de cette hypothèse, il a été observé que :

- les jours où la péche est infructueuse au pied de l'usine (sortie des turbines), elle l'est aussi en tout point du canal de fuite, du moins jusqu'à $1 \mathrm{~km}$ en aval de l'usine où nous avons eu l'occasion de pêcher : 
- s'il y avait accumulation massive, la première heure de pêche le matin et l'après-midi devrait être considérablement plus productive. Or, ceci n'est pas du tout le cas l'après-midi. Le matin, la première heure $(8 \mathrm{~h}$ a $9 \mathrm{~h}$ ) donne des prises seulement $24 \%$ plus abondantes que celles des autres heures de la matinee.

Il existe donc tout au plus une faible concentration aux premières heures du jour mais il n'existe pas d'accumulation de l'effectif d'un jour à l'autre.

Des pêches effectuées la nuit ont toujours été peu productives, ce qui confirmerait les observations de Talbot (1953) et Weaver et al. (1972), selon lesquelles les aloses interrompraient leur migration la nuit.

Les résultats traduisent donc des mouvements de va-et-vient essentiellement diurnes. Les jours où les aloses ne se déplacent pas, on peut supposer que les migrateurs sont en attente sur des aires de repli. Les pêcheurs au "coule" de cette portion de fleuve situent ces zones de repli dans les eaux profondes de l'aval du canal et de la Garonne. Le principal problème est donc de rechercher les facteurs pouvant influencer la fréquence des mouvements d'aloses dans le canal.

\section{4. - Facteurs influant sur la fréquentation de l'usine}

\subsection{1. - Facteur thermique}

A l'examen des résultats, le principal facteur est la température (Tableaux I, II, III). En début de saison (mai), la migration est toujours très réduite lorsque l'eau est à une température inférieure à $16^{\circ} \mathrm{C}$. En 1981 et 1983 , il a fallu attendre le $1^{\text {er }}$ juin pour observer un réchauffement de l'eau au-dessus de $6^{\circ} \mathrm{C}$, coïncidant avec l'arrivée massive des aloses. En 1982, la température était d'emblée de $18^{\circ} \mathrm{C}$ et la migration fut abondante des les premiers jours de pèche.

Hormis ce seuil thermique bien connu des pêcheurs et decrit dans d'autres sites (seuil de $12^{\circ} \mathrm{C}$ pour l'alose du Rhône (Rameyre \& al. 1976); seuil de $16^{\circ} \mathrm{C}$ pour l'alose américaine (Leggett \& Whit. ney 1972), les résultats apportent une information originale : passé le seuil, le sens de la variation thermique est déterminant ; les phases de réchauffement créent des affluences d'aloses et les phases de refroidissement (même si elles n'aboutissent pas à des températures inférieures au seuil) diminuent la fréquence des remontées. Ainsi, en 1983, le refroidissement de 22 à $17^{\circ} \mathrm{C}$ constaté en fin juin réduit les captures alors qu'en mai ces températures permettent le démarrage de la migration (fig. 4).

Il est difficile de réaliser une étude quantitative de l'effet de température sur la migration des aloses car l'influence de ce facteur est différente selon qu'on est en phase de début de migration ou en phase de déclin de migration.

Ainsi, en 1981, si on considère les 28 premiers jours de péche, la corrélation entre les prises et la température est hautement significative $(r=0.815)$ tandis que la même année, pour l'ensemble de la campagne, la relation prises-température n'est pas significative $(r=0.271)$, au seuil de $95 \%$.

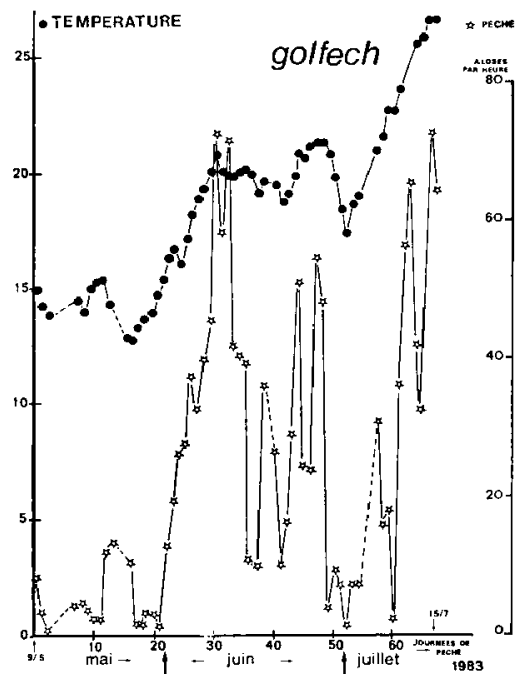

Fig. 4. Représentation de l'efficacité de la pêche en superposition avec l'évolution de la temperature pendant la saison de peche. (Les prises ne deviennent substantielles qu'au-dessus d'un seuil de $16^{\circ} \mathrm{C}$ et, audessus, les phases de réchaulfement sont plus propices à la migration que les phases de re[roidissement). 
La température joue un rôle déterminant en phase d'initiation (seuil) et en phase de developpement de la migration (renforcement des prises en phase de réchauffement). En fin de migration, il y a dissociation complète entre les températures estivales et l'épuisement du stock de migrateurs.

\subsection{2. - Facteur hydraulique}

Après le facteur thermique, l'hydraulique du canal (influencé par le fonctionnement de l'usine) joue un rôle déterminant.

En 1980, 1981 et 1982, l'absence d'aloses, certains jours, restait inexpliquee. En 1983, les enregistrements du débit turbiné à l'usine ont éclairé ce point. A conditions thermiques semblables, les journées de forte migration dans le canal sont celles où le niveau aval reste constant ou mieux, en progressive augmentation dans la journée. Plus fréquemment, les journées où le débit subit une pointe le matin et baisse l'après-midi permettent de bonnes captures le matin et un fléchissement l'après-midi. Ainsi, le flux d'eau joue un rôle déterminant dans l'incitation des a loses à la migration dans le canal artificiel de Golfech.

Les facteurs defavorables à la migration sont les changements de cote subits (changement de turbine...) et les opérations de dégrillage des débris accumulés sur la grille amont (journée du 8 juillet 1983).

Ces résultats ne peuvent être reliés aux observa. tions effectuées sur les rivières: Rameyre \& al. (1976) signalent, sur le Rhône, que la remontée des aloses était favorisée par la baisse des eaux. Aux dires des pêcheurs, il en serait de même en Garonne (Cassou-Leins \& Cassou-Leins 1981, Maudet 1983). Dans le segment artificiel de Golfech, l'augmentation du flux d'eau incite les aloses a aller explorer l'obstacle tandis que les baisses du débit favorisent le repli des aloses.

\subsection{3. - Autres factetirs}

Les résultats ne traduisent aucune influence des facteurs pH et oxygene. La turbidité de l'eau pour. rait jouer un róle non négligeable et une expérimentation sur ce facteur sera programmée ulterieurement.
Pour ce qui concerne les conditions atmosphériques, les pecheurs affirment que le temps lourd et orageux stimule les déplacements des aloses. Cela s'est vérifié en 1983, les 7 et 12 juin, encore qu'en cette période de forte remontée. il est difficile d'imputer l'efficacité de la péche aux conditions météorologiques.

Contrairement à 1981, il n'a été remarqué, ni en 1982 ni en 1983, d'influence du cycle lunaire. Ce facteur, s'il influe, est largement dominé par les facteurs thermique et hydraulique. Les pécheurs at tachent une importance au cycle lunaire sans doute parce que ce facteur influence le comportement de reproduction, la nuit, sur les frayères à aloses. Cet élèment ne semble pas concerner les déplacements diurnes dans le canal artificiel de Golfech.

\section{5 - Marquages}

Il est théoriquement possible d'évaluer une population de poissons par marquage d'un échantillon et par étude du taux de recapture.

Pour les aloses, il est apparu que les techniques de marquage habituelles sont très stressantes et ce r'est qu'en utilisant des marquages de nageoires effectués en maintenant le poisson dans l'eau, qu'on peut mener à bien cette opération.

Toutes les opérations de marquage d'aloses se sont soldées par de très faibles taux de recapture et les résultats sont anecdotiques : 2 recaptures sur les 594 aloses marquées à l'usine en 1983 ! Ce résul. tat est du même ordre de grandeur que celui obtenu par le C.S.P. au niveau de Beauregard en 1982:11 aloses recapturées sur 1217 marquées (Maudet 1983). Même si on marque à plus grande échelle ( 300 aloses marquées à Beauregard le 17 jujn 1983) le taux de recapture reste insignifiant (une alose capturée à Golfech et une alose capturée à Agen).

Les présentes expériences confirment la difficulté de marquer ou de manipuler ces poissons migrateurs et mettent en évidence une perturbation complète du comportement migratoire dès lors que l'animal a été manipulé

\section{6. - Observations sur le comportement grégaire des aloses}

Quelle que soit l'abondance des aloses à Golfech, le " coule » permet d'obtenir très souvent des pri- 
ses multiples entrecoupées de nombreux sondages infructueux. Ceci indique que les aloses se déplacent en petits groupes. Cette observation peut avoir des implications pratiques pour la construction d'échelles à aloses. Elle serait a rapprocher de celles de Cassou-Leins \& Cassou-Leins (1981) qui, à Beauregard, ont observé des aloses en position d'attente devant le barrage et qui passent de manière intermittente, certains jours, en s'engouffrant par groupes dans le chemin ouvert par un leader.

\section{Remerciements}

Ce travajl a été réalisé avec l'aide financière et le consentement d'ELECTRICITE de FRANCE, Région Equipement Marseille.

Les auteurs tiennent aussi à remercier Monsieur Lanies, pécheur professionnel, puur son admirable dévouement et son efficacité.

\section{Travaux cités}

Carscadden (J.E.) \& Leggett (W.C.). 1975. - Meristic difference in spawning populatiıns of american shad, Alosa sapidissima: evidence for homitug tu tributaries in the St-John river. New Brunswick. J. Fish. K'* Bd. Can., 32:653-660.

Cassou-Leins (F.) \& Casseu Leins (J.J.). 1981. - Recherches sur la biologie et l'halieutique des migrateurs de la Garonne et principalement de l'alose Alosa alosa L. These 3 cycle. I.N.P. Toulouse, $382 \mathrm{p}$

Chittinden (M.E.) Jr. 1976. - Weight loss, mortality, feeding and duration of residence of adult american shad (Alosa sapidissima) in freshwater. Fish. Bull., 74 (1): 151-157.

C.T.E.G.R.E.F. 1979. - Etude halieutique de l'estuaire de la Gironde, Groupement de Bordeaux, $189 \mathrm{p}$.
Dautrey (R.) \& Lartigue (J.P.). 1983. - Recherches sur la migration des aloses (Alosa alosa $L$.) et des truites de mer (Salmo rrusta) en Garonne (site de Golfech). Thèse $3^{*}$ cycle, I.N.P. Toulouse. $212 \mathrm{p}$.

Douchement (C.) 1981. - Les aloses des fleuves français, Alosa fallax Lacépède 1803 et Alosa alosa Linné 1578, biométrie écobiologie: autonomie des pupulations. These 3 eycle, Universite des Sciences et Techniques du Languedoc, Montpellier, 377 p.

Leggett (W.C.). 1976. - The american shat (Alosa sapidissima) with special reference to $\mathrm{its}$ migration and population dynamics in the Connecticut river. In: The Connecticut river ecological study, Monography $n^{\circ} /$ by the American Fisheries Society: 169-225.

Legget (W.C.) \& Whitney (R.R.), 1972. - Water temperature and the migrations of american shad. Fish. Invest. Lond. Serv., 2-4 (2) : 1.32 .

Marcy (B.C.) Jr. 1976. - Early life history studies of american shad in the lower Connecticut river and the effects of the Connecticut Yankee plant. In: The Connecticul river ecological siudy. Monography $n^{\circ} /$ by the American Fisheries Society : 141-168.

Maudet (A.). 1983. - L'alose à Agen. Rapport de stage 3e année, E.N.S.A. Toulouse, 129 p.

Quignard (J.P.) \& Kartas (F.). 1977. - Les aloses feintes Alosa fallax (Lacépede 1803), poissons clupéiformes de l'Atlantique Nord-Ouest et de la Méditerranée. Etude des caractères numériques. Bull. Mus. nat. Hist. Nat, 350 : $1241-1256$.

Rarneyre (L.), Kiener (A.), Spillmann (C.P.) \& Biousse (J.) 1976. Aspects de la biologie de l'alose du Rhóne. Péches et difficultés croissantes de ses migrations, Bull. Fr. Pisc., 263 : $50-56$.

Saila (S.B.), Polgar (T.T.), Sheeky (D.J.) \& Flowers (J.M.). 1972 . Correlations between alewife activity and environmental variables at a fishways. Trans. Am. Fish. Soc., 4 : 583-594.

Talbot (G.B.). 1963. - Passage of the shad at the Bonreville fishways. U.S. Fish. Wildl Serv., Spec. Sci. Rep. Fish., n ${ }^{\circ} 94,30$ p.

Walburg (C.H.) \& Nichols (P.R.). 1967. - Biology and management of the american shad and status of the fisheries atlantic coast of the United States. U.S. Fish. Wildl Serv., Spec. Sci. Rep. no 550, $105 \mathrm{P}$.

Weaver (C.R.). 1963. - Influence of water velocity upon orientation and performance of aulult migrat ory salmonids. U.S. Fish. Wild]. Serv. Fish. Bull., 63 (1), 97.121.

Weaver (C.R.), Thompson (C.C.) \& Ossiander (F.J.). 1972. - Evaluation of fish passage in the vertical slot regulating section of the suuth shore ladder at John Day dam. Nai. Mar. Fish. Serv., Final report, $58 \mathrm{p}$. 